\title{
A Case of Intraocular Erosion and Intrusion by an Arruga Suture
}

\author{
Takakuni Kitagaki $^{a}$ Seita Morishita ${ }^{a}$ Ryohsuke Kohmoto ${ }^{a}$ \\ Masanori Fukumoto $^{a}$ Hiroyuki Suzuki $^{a}$ Takaki Sato $^{a}$ \\ Takatoshi Kobayashi $^{a}$ Teruyo Kida $^{a}$ Masayuki Nakajimab \\ Tsunehiko Ikeda ${ }^{a}$ \\ ${ }^{a}$ Department of Ophthalmology, Osaka Medical College, Takatsuki, and \\ ${ }^{b}$ Nakajina Eye Clinic, Kyoto, Japan
}

\section{Key Words}

Arruga suture $\cdot$ Scleral buckling surgery $\cdot$ Retinal detachment $\cdot$ Iritis

\begin{abstract}
Purpose: To report a case of intraocular erosion and intrusion by an Arruga suture. Case Report: This study involved a 62-year-old male who had undergone scleral buckling surgery 40 or more years ago at another hospital for rhegmatogenous retinal detachment, as well as trabeculectomy 20 years ago for primary open-angle glaucoma, in his left eye at the same hospital. However, he recently became aware of blurred vision in that eye. Upon examination, iritis was observed in the anterior portion of his left eye, as well as a great number of pigment cell keratoprecipitates on the posterior surface of the cornea. In the ocular fundus, extensive atrophy of the retinal pigment epithelium and partial hyperpigmentation was observed, accompanied by subretinal strands, yet the retina remained attached. Around the entire peripheral area of the retina we observed a ring-shaped protrusion, but we also saw a shiny, filamentous material in the vitreous cavity that penetrated the sclera and choroid, completely extending from the 4- to 8-o'clock position of the scleral buckle protrusion. To treat the iritis in the patient's left eye, we began to administrate low-concentration steroid eye drops, after which the inflammation disappeared. Conclusion: In this study, we experienced a case of intraocular erosion and intrusion by an Arruga suture, and opted to treat the patient with steroid eye drops and conservative follow-up observations unless retinal redetachment and/or vitreous hemorrhage occurs.


Kitagaki et al.: A Case of Intraocular Erosion and Intrusion by an Arruga Suture

\section{Introduction}

Prior to the use of silicone materials becoming popular for scleral buckling surgery, encircling procedures which utilized a thread known as 'Arruga suture' were performed [1-6]. The procedure was simple and widely practiced in European countries, yet it has been used in only a small fraction of medical facilities in Japan, since the suture thread used for the procedure reportedly can erode the sclera and the choroid, lift the retina into a tent-like shape, and cause complications such as exposure into the vitreous cavity [7, 8]. In this study, we present the case of a patient who experienced intraocular erosion and intrusion by an Arruga suture.

\section{Case Report}

This study involved a 62-year-old male who had undergone scleral buckling surgery 40 or more years ago for rhegmatogenous retinal detachment, as well as trabeculectomy 20 years ago for primary open-angle glaucoma in his left eye at another hospital. He presented to our hospital complaining of blurred vision in that eye. The patient reported that although the previous retinal reattachment surgery had resulted in a favorable outcome, he had experienced persistent conjunctival hyperemia, conjunctival edema, and low levels of ptosis during the subsequent recovery period. He reported that he had recently become aware of blurred vision in his left eye and subsequently consulted a local doctor. Upon examination at that facility, iritis and proliferating changes in the fundus had been identified in that eye, and he had been referred to our hospital.

Upon examination, his visual acuity was $1.0 \times \mathrm{S}-3.5 \mathrm{D} \mathrm{C}-1.5 \mathrm{D} \mathrm{A} \times 30^{\circ} \mathrm{OD}$ and $0.5 \mathrm{p} \times \mathrm{S}+$ 2.0D C-2.25D A $\times 160^{\circ} \mathrm{OS}$, and his intraocular pressure was $20 \mathrm{~mm} \mathrm{Hg}$ OD and $11 \mathrm{~mm} \mathrm{Hg}$ OS. Iritis was observed in the anterior chamber of the left eye, as well as a great number of pigment cell keratoprecipitates on the posterior surface of the cornea (fig. 1). Minor cataracts were observed beneath the posterior capsule, and mydriasis was somewhat poor. In the ocular fundus of the left eye, extensive atrophy of the retinal pigment epithelium and partial hyperpigmentation were observed, accompanied by subretinal strands, yet the retina remained reattached. Around the entire peripheral area of the retina we observed a high, ring-shaped protrusion, but we also saw a shiny, filamentous material in the vitreous cavity that penetrated the sclera and choroid, completely extending from the 4- to 8-o'clock position of the protrusion (fig. 2). During a B-mode ultrasound scan, we observed a highluminance image on the edge of the buckle protrusion with an acoustic shadow (fig. 3). We also observed slight opacity and inflammatory cells in the vitreous cavity. Thus, we diagnosed this patient as a case of intraocular erosion and intrusion by an Arruga suture, and for the iritis in the left eye we began to administrate low-concentration steroid eye drops, after which the inflammation disappeared. A previous doctor had performed a trabeculectomy on the left eye 20 years previously to treat primary open-angle glaucoma, and we recognized a filtration bleb in the upper part of the corneal limbus, but we saw no particular abnormalities in the middle optic media or fundus in the right eye.

\section{Discussion}

The Supramid suture used in an Arruga suture is composed of a non-absorbable pseudomonofilament with a polyamide core. Arruga sutures are narrower than silicone sutures, 
Kitagaki et al.: A Case of Intraocular Erosion and Intrusion by an Arruga Suture

and although the constriction per unit area is high, they have poor elasticity; thus, it is believed that they can easily erode the eyeball wall [3]. Today, silicone is generally used as the buckle material for retinal detachment surgery, and the Arruga suture is seldom, if ever, used. The Arruga suture in this case was about $2 \mathrm{~mm}$ in diameter and had a slight luster on its surface [2]. Ocular complications related to Arruga sutures have sometimes been reported in the past, but recent reports are extremely rare. Manson [9] described cases following Arruga suture surgery that included chronic conjunctival hyperemia, conjunctival edema, iritis, posterior iris synechia, and ptosis, etc., as 'string syndrome,' yet we believe that these are also mild forms of this complication.

We speculate that the conjunctivitis and iritis etc. observed in patients who have undergone Arruga suture surgery are caused by a circulatory disturbance of the choroid, brought on by constriction of the eyeball by the Arruga suture. In this case as well, we observed slight degrees of conjunctival hyperemia, conjunctival edema, ptosis, and iritis, and we believe that these symptoms had persisted since a previous period. We believe that in cases like this that include iritis and low levels of vitreous opacity, or Arruga suture exposure into the vitreous cavity without retinal redetachment, conservative treatment and observation are indicated. We speculated that fundus hyperpigmentation and retinal pigment epithelial atrophy were caused by chronic intraocular inflammation due to the Arruga suture. Vitreous surgery is also indicated, but only in cases where complications remarkably affect the patient's visual function. We believe that surgical indications for ocular complications by an Arruga suture include cases where the Arruga suture intraocular erosions cause recurrences of rhegmatogenous retinal detachment, non-rhegmatogenous lifted-form detachments extending to the macula, or choroidal or vitreous hemorrhage caused by the Arruga suture. Previously, surgical treatment for these complications involved external removal of the Arruga suture. In cases where the Arruga suture has become adhered to the sclera and Tenon's capsule, removal is difficult and includes the danger of intraocular fluid leakage and subsequent scleral rupture. Due to the recent advancements in vitreous surgery, it has now become relatively safe to operate via the transvitreal procedure, and it makes sense to perform treatment from within the vitreous cavity. We believe that using vitreous surgical scissors and cutters allows for the removal of an Arruga suture which is exposed to the vitreous cavity, and endophotocoagulation of the perforated part can generally be performed. In some cases appropriate scleral patch grafts may be necessary to cover defects after the partial removal of the Arruga suture.

In conclusion, although it has been reported that buckle materials such as polyethylene rod erode the sclera in the same manner as Arruga sutures [10], it is important to be aware that similar complications can arise when hard buckling materials are used.

\section{Acknowledgements}

The authors wish to thank John Bush for editing the paper.

\section{Statement of Ethics}

The authors have no ethical conflicts to disclose. 
Kitagaki et al.: A Case of Intraocular Erosion and Intrusion by an Arruga Suture

\section{Disclosure Statement}

The authors have no conflicts of interest to report.

\section{References}

1 Arruga H: Retinal detachment operations. Bibl Ophthalmol 1957;12:443-447.

-2 Dittmann M, Graeber W: Operation of retinal detachment with the Arruga suture. Klin Monbl Augenheilkd 1963;143:647-653.

-3 Algvere P: Reinforced episcleral cerclage in retinal detachment. A note on surgical technique. Acta Ophthalmol (Copenh) 1977;55:591-596.

4 McAuley FD: The Arruga suture for retinal detachment 17 years on. Trans Ophthalmol Soc UK 1975;95: 165-166.

5 Mortada A, el-Shewy T, Eleinein GA: Combined lamellar scleral resection overlaying the retinal tear and Arruga encircling thread in retinal detachment surgery. Bull Ophthalmol Soc Egypt 1967;60:107-112.

6 Somerset EJ: Experiences with Arruga suture. Trans Ophthalmol Soc UK 1966;86:735-742.

7 Greven CM, Kurup SK, Jacklin HN: Intraocular erosion and intrusion of an Arruga encircling suture. Retina 2011;31:1437-1438.

-8 D'Hermies F, Korobelnik JF, Chauvaud D, Pouliquen Y, Parel JM, Renard G: Scleral and episcleral histological changes related to encircling explants in 20 eyes. Acta Ophthalmol Scand 1999;77:279-285.

-9 Manson N: The 'string syndrome' seen as a complication of Arruga cerclage suture. Br J Ophthalmol 1964;48:70-74.

10 Doyle E, Georgalas I, Sullivan P, Laidlaw D: Intrusion of an encircling band with recurrent retinal detachment: surgical approach. Br J Ophthalmol (Video Report) 2006.

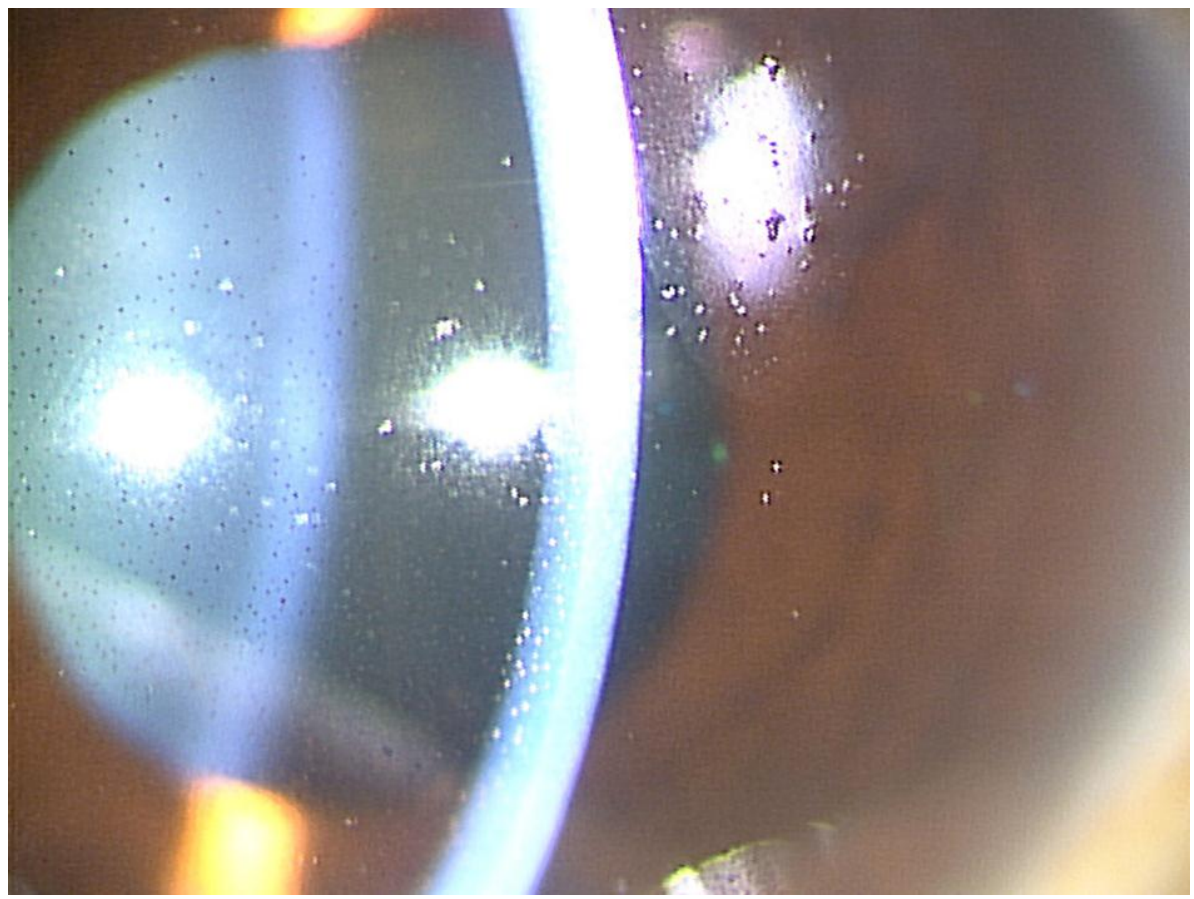

Fig. 1. Slit-lamp photograph of the patient's left eye. Mild iritis and a large number of pigment cell keratoprecipitates can be seen. 
Kitagaki et al.: A Case of Intraocular Erosion and Intrusion by an Arruga Suture

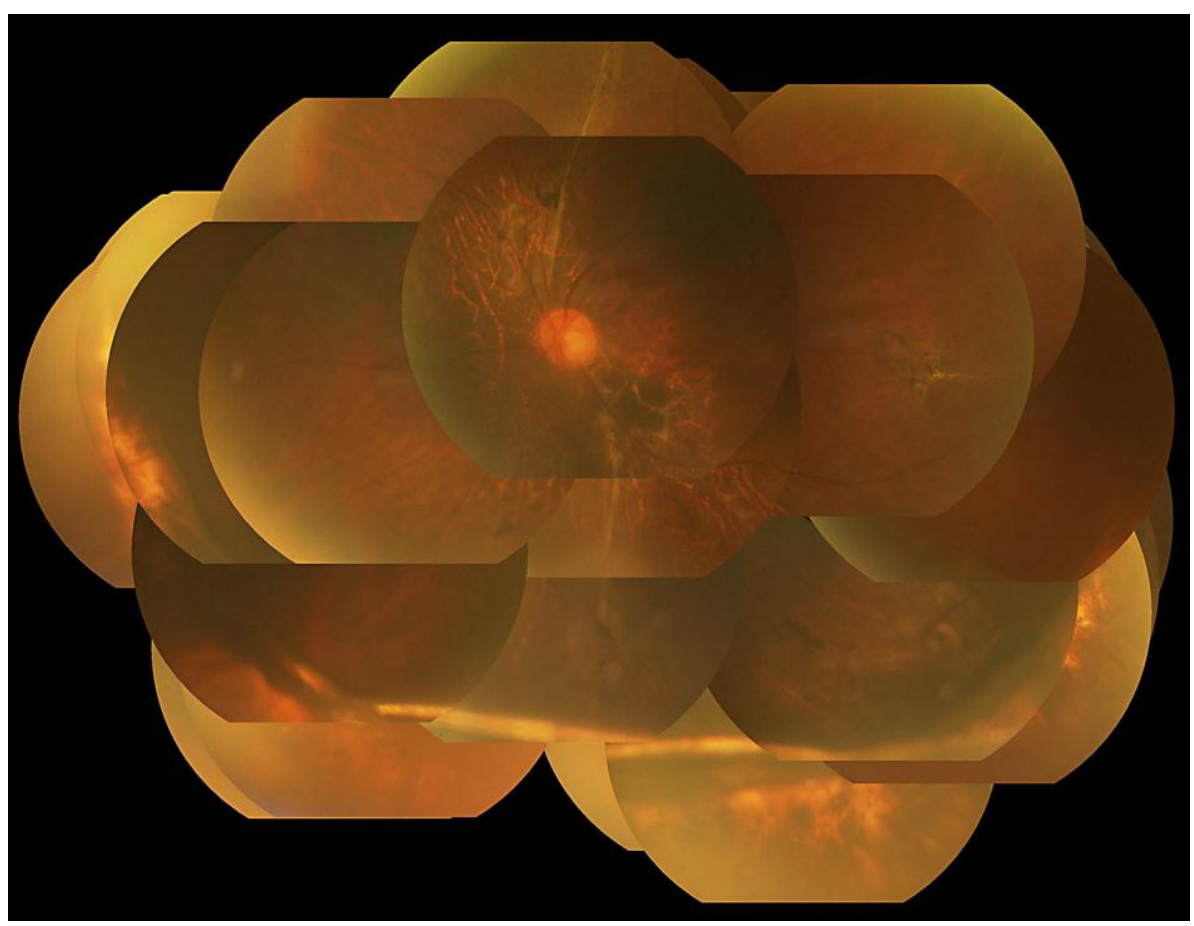

Fig. 2. Fundus photograph of the patient's left eye. A shiny, filamentous material in the vitreous cavity penetrating the sclera and choroid completely, extending from the 4- to 8-o'clock position, can be seen.

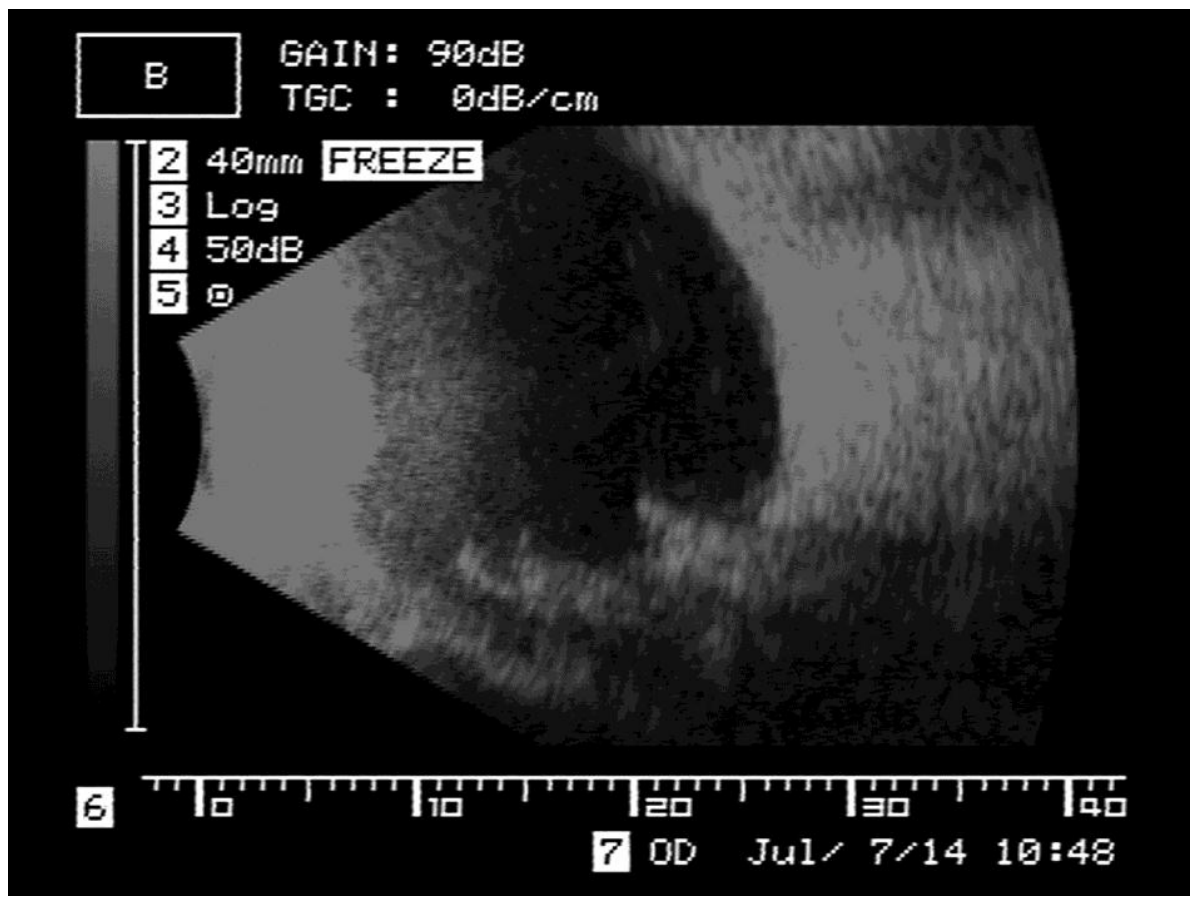

Fig. 3. B-mode ultrasound scan of the patient's left eye. A high-luminance image on the edge of the buckle protrusion with an acoustic shadow can be seen. 